\title{
ON MODULAR EQUATIONS.
}

BY PROFESSOR JAMES PIERPONT.

(Read at the March Meeting of the Society, 1897.)

The theory of the equations of transformation has been put in an entirely new light and very essentially improved by H. Weber's paper "Zur Theorie der Elliptischen Functionen.* His starting point is the solution of the equation for the division of the periods making a systematic use of the Galoisian theory of equations. From this standpoint it is not the modular equation

$$
M(v, u)=0
$$

with coefficients rational in $u={ }^{4} \sqrt{k}$ that we are led to consider but the equation

$$
T(y, x)=0
$$

whose coefficients are rational in $x=k^{2}$ and whose roots are the $n+1$ values of

$$
\prod_{p=1}^{m} \frac{\mathrm{cn}}{\mathrm{dn}}\left(p \frac{4 \lambda K+4 \mu i K^{\prime}}{n}\right) \quad \begin{aligned}
& \lambda, \mu=0,1 \cdots n-1 \\
& \lambda=\mu=0 \text { excluded }
\end{aligned}
$$

Here for simplicity we take $n=2 m+1$, an odd prime.

Let us see how the coefficients of this equation can be calculated. As the roots of $T$ differ from those of $M$ only by the factor $u^{-n}$, the $T$ equations could be derived from the modular equations $M=0$ on setting $v=u^{n} y$. But the methods given to compute the modular equations compel us-as far as I have been able to consult the literature-to pass from our domain of rationality $R(x)$ to that of $R(u)$, and this from our standpoint is certainly objectionable unless necessary. To show that this is not so is the first object of the present paper. Again, the methods given to calculate $M=0$ are made-as far as I know-to depend upon the transformation theory of Hermite's function $u=\varphi(\tau)$. I propose to show as second object that we can calculate our $T$ equation without leaving the $\vartheta$ 's. A considerable simplification is thus obtained. But, having simplified the calculation of $T$ so far, I have been tempted to go one step farther and show how we may arrive at Weber's equations

\footnotetext{
* Acta Mathematica, vol. 6, p. 329. Also his book Elliptische Func-
} tionen und Algebraische Zahlen, Braunschweig, 1891. 
of transformation without the Galoisian Theory; this is my third object. In this way a tolerably simple and compact theory for calculating the $T$ equation is given.

1.

We show first how we may arrive at Weber's Equations. The multiplication theorem for

$$
\operatorname{sn} u, \operatorname{cn} u, \operatorname{dn} u
$$

tells us three things that are of importance here :

$1^{\circ}$ the $n^{2}-1$ quantities

$$
x_{\lambda, \mu}=\operatorname{sn} \frac{\lambda 4 K+\mu 4 i K^{\prime}}{n}=\operatorname{sn} w_{\lambda, \mu}
$$

are roots of an equation

$$
P(x ; x)=0
$$

whose coefficients are rational in $x$, $2^{\circ}$ the quantities

$$
\operatorname{cn} w_{\lambda, \mu}, \quad \operatorname{dn} w_{\lambda, \mu}
$$

are rational* in $x_{\lambda, \mu}^{2}$

$3^{\circ}$

$$
x_{p \lambda, p \mu}=\theta_{p}\left(x_{\lambda, \mu}\right)
$$

where $\theta_{p}$ is a rational function independent of $\lambda, \mu$.

This last remark shows us that the roots of $P=0$ can be arranged in the array $\dagger$

$$
\begin{aligned}
& x_{1,0} \quad \theta x_{1,0} \quad \theta^{2} x_{1,0} \quad \cdots \cdots \theta^{n-2} x_{1,0} \\
& x_{0,1} \quad \theta x_{0,1} \quad \theta^{2} x_{0,1} \quad \cdots \cdots \cdot \theta^{n-2} x_{0,1} \\
& x_{n-1,1} \quad \theta x_{n-1,1} \quad \theta^{2} x_{n-1,1} \cdots \cdots \theta^{n-2} x_{n-1,1}
\end{aligned}
$$

For let $g$ be a primitive congruence root of $n$, then $3^{\circ}$ shows that if

then

$$
x_{g \lambda, g \mu}=\theta x_{\lambda, \mu}
$$

$$
x_{g^{2} \lambda, g^{2} \mu}=\theta x_{g \lambda, g \mu}=\theta \cdot \theta x_{\lambda, \mu}=\theta^{2} x_{\lambda, \mu} \text { etc. }
$$

*We take from now on $R(\kappa)$ for domain of rationality. For a simple explanation of this term see my paper in the Bulletin for December, 1895 , p. 81.

†Cf. A ABLL. Oeuvres, vol. I., p. 478. Sur une classe particulière d'équations, etc. 
Let now $t_{\infty}$ be a rational symmetric function of the roots of the first row of $A$

$$
t_{\infty}=f\left(x_{1,0} \quad \theta x_{1,0} \cdots \cdots \cdot \theta^{n-2} x_{1,0}\right)=F\left(x_{1,0}\right)
$$

where $F$ is a rational function. On replacing here $x_{1,0}$ by

$$
x_{1,0} \quad x_{0,1} \quad x_{1,1} \cdots \cdots x_{n-1,1}
$$

$t_{\infty}$ goes over into quantities which we denote respectively by

$$
t_{\infty}, t_{0}, t_{1} \cdots t_{n-1}
$$

These are roots of an equation of degree $n+1$

$$
W(t, x)=0
$$

whose coefficients lie in $R(x)$. In fact if

$$
\begin{gathered}
\iota \equiv \frac{\lambda}{\mu} \quad \bmod n \\
t_{\iota}=F\left(x_{\lambda, \mu}\right)=F\left(\theta x_{\lambda, \mu}\right)=\cdots=F\left(\theta^{n-2} x_{\lambda, \mu}\right) \\
\iota=\infty, 0,1 \cdots n-1
\end{gathered}
$$

Hence

$$
t_{\iota}^{p}=\frac{1}{n+1} \sum_{h} F^{p}\left(\theta^{h} x_{\lambda, \mu}\right) \quad h=0, \cdots n-2 .
$$

and thus

$$
\sum_{\iota} t_{\imath}^{p}
$$

is a symmetric function of the roots of $P(x, x)=0$ and hence lies in $R(x)$; hence etc.

The equations $W=0$ are Weber's equations of transformation. That our $T$ equation belongs to this class is seen thus. We observed at the commencement of this paragraph that

$$
\operatorname{cn} w_{\lambda, \mu}, \operatorname{dn} w_{\lambda, \mu}
$$

were rational in $x_{\lambda, \mu}^{2} ;$ hence

$$
y_{\infty}=\prod_{p=1}^{m} \frac{\mathrm{cn}}{\mathrm{dn}}\left(p w_{1,0}\right)
$$

is a rational symmetric function of

and hence of

$$
x_{1,0}^{2} \quad x_{2,0}^{2} \cdots x_{m, 0}^{2}
$$$$
x_{1,0} \quad x_{2,0} \quad \cdots \cdots \cdots x_{n-1,0}
$$

that is of the roots in the first row of $A$. 
2.

Having shown that the $n+1$ quantities

(1) $y_{\infty}=\Pi \frac{\mathrm{cn}}{\mathrm{dn}}\left(p \frac{4 K}{n}\right), \quad y_{a}=\Pi \frac{\mathrm{cn}}{\mathrm{dn}}\left(p \frac{16 a 4 K+4 i K^{\prime}}{n}\right)$

$$
\begin{aligned}
& p=1,2 \ldots m \\
& a=0,1 \ldots n-1
\end{aligned}
$$

are roots of a rational equation

(2) $\quad T(y, x)=f_{0}(x) y^{n+1}+f_{1}(x) y^{n}+\cdots+f_{n+1}(x)=0$

we turn to the calculation of its coefficients.

To arrive at our goal it is necessary to know

(a) a superior limit of $x$ in $I$

( $\beta$ ) how to develop the roots of $T$ into $q$-series.

Having these facts, the coefficients may be obtained in either of the two ways indicated by Sohnke.*

Let us consider $(\beta)$ first. Setting as usual

$$
\sqrt{k}=\frac{\vartheta_{2}(0 \mid \tau)}{\vartheta_{3}(0 \mid \tau)}=\sqrt{k(\tau)}
$$

the transformation theory of the $\vartheta$ 's gives us

$$
\begin{gathered}
\sqrt{k(n \tau)}=(\sqrt{k})^{n} \prod_{p=1}^{m} \frac{\mathrm{cn}^{2}}{\mathrm{dn}^{2}}\left(p \frac{4 K}{n}\right)=(\sqrt{k})^{n} y_{\infty}^{2} \\
\sqrt{k\left(\frac{\tau+16 a}{n}\right)}=(\sqrt{k})^{n} \prod_{p=1}^{m} \frac{\mathrm{cn}^{2}}{\mathrm{dn}^{2}}\left(p \frac{16 a 4 K+4 i K^{\prime}}{n}\right)= \\
(\sqrt{k})^{n} y_{a}^{2}
\end{gathered}
$$

Hence extracting the square roots of these equations and setting for simplicity

$$
u=\varphi(\tau)=\sqrt{2} q^{\frac{1}{8}} \prod_{\nu=1}^{\infty} \frac{1+q^{2 \nu}}{1+q^{2 \nu-1}}
$$

we have

$$
\begin{gathered}
y_{\infty}= \pm \frac{\varphi(n \tau)}{\varphi^{n}(\tau)} \\
y_{a}= \pm \frac{\varphi(\tau+16 a)}{\varphi^{n}(\tau)}
\end{gathered}
$$

* Crelle, vol. 16, p. 97, Aequationes modulares pro transformatione functionum ellipticarum. 
The sign of $y_{\infty}$ we get by observing that if $\tau=r+i s$

$$
\lim _{s=\infty} \frac{\vartheta_{2}(u)}{\sqrt{k}}=2 \cos \pi v, \quad \lim _{s=\infty} \vartheta_{s}(u)=1
$$

and we have

$$
\lim _{s=\infty} y_{\infty}=\lim \Pi \frac{1}{\sqrt{k}} \frac{\vartheta_{2}\left(\frac{2 p}{n}\right)}{\vartheta_{3}\left(\frac{2 p}{n}\right)}=\Pi \cos \frac{2 \pi p}{n}=\frac{\gamma}{2^{m}}
$$

where

$$
\gamma=\left(\frac{2}{n}\right)=(-1)^{\frac{n^{2}-1}{8}}
$$

Hence

$$
y_{\infty}=\gamma \frac{\varphi(n \tau)}{\varphi^{n}(\tau)}
$$

The sign of $y_{0}$ is at once obtained by observing that for $\tau=i s, \vartheta_{2}(u), \vartheta_{3}(v)$ are real and positive when $v$ is purely imaginary.

Thus

$$
y_{0}=\frac{\varphi\left(\frac{\tau}{n}\right)}{\varphi^{n}(\tau)} \text {. }
$$

The sign of the other roots we get by using the identity

$$
T\left[y_{0}(\tau), x(\tau)\right]=T\left[\frac{\varphi\left(\frac{\tau}{n}\right)}{\varphi^{n}(\tau)}, x(\tau)\right]=0
$$

If we replace here

$$
\tau \text { by } \tau+16 a
$$

equation (3) shows that

$$
\varphi(\tau+16 a)=e^{\frac{\pi i}{8} 16 a} \varphi(\tau)=\varphi(\tau)
$$

whence (4) becomes

$$
T\left[\frac{\varphi\left(\frac{\tau+16 a}{n}\right)}{\varphi^{n}(\tau)}, x\right]=T\left(y_{a}, x\right)=0
$$

which shows that

$$
y_{a}=\frac{\varphi\left(\frac{\tau+16 a}{n}\right)}{\varphi^{n}(\tau)}
$$


is a root. As these are all distinct we have now our roots expressed as infinite $q$-products which may he converted into $q$-series either by multiplying and dividing out or by making use of the transformation theory of the find

$$
\begin{gathered}
y_{\infty}=r 2^{-m}\left[1+a_{1} q+a_{2} q^{2}+\cdots\right] \\
y_{a}=2^{-m} q^{-\frac{r}{n} \varepsilon^{a}}\left[1+\varepsilon^{8 a} b_{1} q^{\frac{1}{n}}+\varepsilon^{16 a} b_{2} q^{\frac{2}{n}}+\cdots\right] \\
\varepsilon=e^{2 \pi i} \quad r=\frac{n^{2}-1}{8}
\end{gathered}
$$

3.

We have now three different expressions for the roots of our $T$ equation from which we can at once draw some simple conclusions regarding the coefficients $f(x)$ of (2). In the first place the coefficient $f_{0}(x)$ must reduce to a monomial, since roots of

$$
f_{0}(x)=0
$$

are the infinities of the $y$ 's and these, as we now can see, are infinite only at $x=0$. We can thus write $T=0$ in the form

where

$$
y^{n+1}+g_{1}(x) y^{n}+g_{2}(x) y^{n-1}+\cdots+g_{n+1}(x)=0
$$

$$
g_{s}(\kappa)=\frac{a_{s, 0}+a_{s, 1} \chi+\cdots+a_{s, m_{s}} x^{m_{s}}}{x^{r_{s}}}
$$

The developments (5) in connection with Newton's formulæ

$$
\begin{aligned}
& g_{1}+s_{1}=0 \\
& 2 g_{2}+s_{1} g_{1}+s_{2}=0 \\
& 3 g_{3}+3_{1} g_{2}+s_{2} g_{1}+s_{3}=0
\end{aligned}
$$

give us superior limits of the $r$ 's. Consider first $r_{n+1}$ : Evidently

$$
\lim _{\kappa=0} x^{r} n+1\left(y_{\infty} \cdot y_{0} \cdots y_{n-1}\right)=a_{n+1,0}
$$

and (5) shows that this requires that

$$
r_{n+1}=r \quad a_{n+1,0}=r
$$

Consider now $r_{1}$; we have

$$
-s_{1}=g_{1}
$$

*Sohnke loc. cit. gives $\varphi(\tau)$ to the 26th power of $q$; also the first 20 powers of $\varphi$ are given. 
hence the order of $g_{1}$ for $x=0$ is that of $s_{1}$, or since $y_{\infty}$ is finite that of

$$
y_{0}+y_{1}+y_{2}+\cdot+y_{n-1}
$$

But (5) shows that this sum is

$$
\begin{gathered}
q^{-\frac{r}{n}}\left[b_{0} \sum \varepsilon^{n}+b_{1} q^{\frac{1}{n}} \sum \varepsilon^{h(8+1)}+b_{2} q^{\frac{2}{n}} \sum \varepsilon^{h(2,8+1)}+\cdots\right] \\
h=0,1 \cdots n-1
\end{gathered}
$$

As $\sum_{h} \varepsilon^{h_{\rho}}$ is $n$ or 0 according as $\rho$ is or is not $\equiv 0 \bmod$. $n$, we see that if $\nu_{1}$ is the smallest positive root of

$$
8 \nu+1 \equiv 0 \text { mod. } n
$$

the order of $s_{1}$ is

$$
\sigma_{1}=\frac{r-\nu_{1}}{n}
$$

In regard to the other exponents this method affords only a superior limit.* The orders

of

$$
\begin{array}{ll}
\sigma_{2} & \sigma_{3} \cdots \\
s_{2} & s_{3} \cdots
\end{array}
$$

are found from (5) to be

$$
\begin{aligned}
& \sigma_{2}=\frac{2 r-\nu}{n} \quad \text { where } \quad 4 \nu_{2}+1 \equiv 0 \\
& \sigma_{3}=\frac{3 r-\nu_{3}}{n} \quad \cdots \quad 8 \nu_{3}+3 \equiv 0 \\
& \sigma_{4}=\frac{4 r-\nu_{4}}{n} \quad \cdots \quad 2 \nu_{4}+1 \equiv 0 \quad \bmod . n \\
& \sigma_{5}=\frac{5 r-\nu_{5}}{n} \quad \cdots \quad 8 \nu_{5}+5 \equiv 0
\end{aligned}
$$

Where here $\nu$ is the smallest positive root of its corresponding congruence.

Newton's formulæ show us that $r_{2}$ is not superior to the greater of the orders of $s_{2}, s_{1} g_{1}$ etc. In certain cases of frequent occurrence we need consider only the $\sigma$ 's. Thus suppose we had found that the orders of

$$
g_{1} \quad g_{2} \cdots g_{a}
$$

\footnotetext{
* In $\& 8$ we shall see that $r_{n}$ is also determined viz: $r_{n}=r$.
} 
were not greater respectively than

$$
\sigma_{1} \quad \sigma_{2} \cdots \sigma_{\alpha}
$$

then the order of $g_{a+1}$ is not superior to $\sigma_{a+1}$ if

$$
\sigma_{1}+\sigma_{\alpha}, \sigma_{2}+\sigma_{\alpha-1}, \sigma_{3}+\sigma_{a-2}, \cdots
$$

are not greater than $\sigma_{a+1}$. When the $\sigma^{\prime}$ s have this property we say they form a regular sequence.

4.

Another important and very evident property of the $T$ equation we get from the expression of the roots as quotients of the elliptic functions as given by (1). In fact, for $x=1$ we know the elliptic functions degenerate to exponentials and

$$
\lim _{\kappa=0} \frac{\operatorname{cn}(u, x)}{\operatorname{dn}(u, x)}=1
$$

if $u$ is independent of $x$. With us the case is somewhat different ; here

$$
u=p \frac{4 \lambda K+4 \mu i K^{\prime}}{n},
$$

is not independent of $x$ and (6) does not always approach 1 as a limit for then $a_{n+1,0}$ could not be $\gamma=(-1)^{r}$ as we just found but would always be +1 . It is easy to show* on passing to exponentials that

$$
\lim _{\kappa=1} \frac{\mathrm{cn}}{\operatorname{dn}}\left(p \frac{4 \lambda K+4 \mu i K^{\prime}}{n}\right)= \pm 1
$$

according as

Thus

$$
n-4 \lambda p \gtrless 0
$$

$$
T(y, 1)=(y-1)\left(y-(-1)^{r}\right)^{n}
$$

5.

We turn now to problem ( $\alpha$ ) viz., determine a superior limit† of $x$ in $T$. By making use of the property that $T=0$ remains

* Cf. A. EnNiterer : Elliptische Functionen, 2d Ed. 1890, p. 460.

$\dagger$ We remark that a superior limit of $\kappa$ is given at once, for if

$$
f\left(w^{n}, z^{m}\right)=0
$$

is irreducible, $m$ is equal to the sum of the orders of the infinities for the whole $z$ plane. For $T=0$ this is precisely $r$. 
unaltered for certain substitutions we can go much farther and write down the literal part of $T$ pretty closely. To do this we shall make use of the fact that the $W$ equations are irreducible in $R(x)$. From the Galoisian theory this is self-evident; but we can show it here by making use of a principle Weber* has employed in another connection.

In fact let

$$
V(t, x)
$$

be the irreducible factor of $W(t, x)$ admitting $t_{\infty}$ as root. In the identity

$$
V\left[F\left(x_{1,0}(\tau) ; x(\tau)\right]=0\right.
$$

replace $\tau$ by

$$
\tau_{1}=\frac{c-a \tau}{b \tau-\partial}
$$

where

(9) $\quad a \equiv \partial \equiv 1 \quad b \equiv c \equiv 0 \quad \bmod .4 \quad a \partial-b c=1$

Such a substitution leaves the coefficients of $V=0$ unaltered. Let us see how it affects $t_{\infty}$.

The theory of linear transformation of the $\vartheta$ 's gives

$$
\begin{gathered}
\frac{\vartheta_{s}\left(0 \mid \tau_{1}\right)}{\vartheta_{2}\left(0 \mid \tau_{1}\right)} \frac{\vartheta_{1}\left(\nu \mid \tau_{1}\right)}{\vartheta_{0}\left(\nu \mid \tau_{1}\right)}=e^{\frac{2 \pi i}{4}(1-\alpha)} \frac{\vartheta_{3}}{\vartheta_{2}} \cdot \frac{\vartheta_{1}}{\vartheta_{0}}(\nu(\partial-b \tau) \mid \tau) \\
a \equiv \partial \equiv 1 \quad b \equiv c \equiv 0 \quad \bmod 2 \quad a \partial-b c=1 \\
\tau_{1}=\frac{a \tau-c}{\partial-b \tau}
\end{gathered}
$$

But if we restrict the $a, b, c, \partial$ of (11) to satisfy also (9), the exponential factor drops out of (10). If we now set in $(10)$

it gives

$$
v=\frac{2}{n}
$$

$$
x_{1,0}\left(\tau_{1}\right)=x_{\delta,-b}
$$

The identity (7) after the substitution (8) becomes thus

or setting

$$
V\left[F\left(x_{\delta,-b}\right), x\right]=0
$$

$$
\begin{gathered}
a \partial+b \equiv 0 \quad \bmod n \\
V\left(t_{a}, x\right) 0,
\end{gathered}
$$

* Elliptische Functionen und Algebraische Zahlen, p. 221. 
that is $V(t, x)$ admits all the roots of $W=0$ and $W$ is thus irreducible.*

6.

We can now show at once that $T=0$ is unaltered for the substitution

For if in the identity

$$
\left(\begin{array}{ll}
y, & x \\
y^{-1}, & x^{-1}
\end{array}\right)
$$

$$
T\left[y_{\infty}(\tau) ; \chi(\tau)\right]=0
$$

we replace $\tau$ by $\frac{\tau}{1-\tau}$ it goes over into

$$
T\left[y_{\infty}\left(\frac{\tau}{1-\tau}\right), x^{-1}\right]=0
$$

But the theory of the linear transformation of the $\%$ 's gives

$$
\begin{aligned}
y_{\infty}\left(\frac{\tau}{1-\tau}\right) & =\left(\sqrt{k\left(\frac{\tau}{1-\tau}\right)}\right)^{-m} \Pi \frac{\vartheta_{2}}{\vartheta_{3}}\left(p \cdot \frac{2}{n} \mid \frac{\tau}{1-\tau}\right) \\
= & (\sqrt{k})^{m} \Pi \frac{\vartheta_{3}}{\vartheta_{2}}\left(\frac{2 p(1-\tau)}{n}\right) \quad p=1,2 \cdots m \\
= & \Pi \frac{\mathrm{dn}}{\mathrm{cn}}\left(p \cdot \frac{4 K-4 i K^{\prime}}{n}\right) \\
= & y_{a}^{-1}
\end{aligned}
$$

if

$$
16 \alpha+1 \equiv 0 \quad \bmod n .
$$

Hence (13) becomes

that is the equation

$$
I\left[y_{\alpha}^{-1}, x^{-1}\right]=0
$$

$$
T\left(y^{-1}, x^{-1}\right)=0
$$

of degree $n+1$ has a root in common with the irreducible equation

$$
T(y, x)=0
$$

and is thus identical with it.

\footnotetext{
* It is to be expressly remarked that this reasoning applies equally well to the domain formed by $\kappa$ and any constants $a, \beta, \gamma \ldots$ independent of $\tau$.
} 
Let us see how this helps us to determine the literal part of $T=0$. Apply (12) to $\left(2^{a}\right)$ and make in the resulting equation the coefficient of $y^{n+1}$ unity. The coefficients of the various powers of $y$ in the two equations must be identical. This shows at once that the absolute term must reduce to a monomial and thus its form is definitely

$$
g_{n+1}(x)=\frac{r}{x^{r}}
$$

Let us look at the term

$$
\alpha_{\lambda, \mu} \frac{y^{\lambda}}{\chi^{\mu}}
$$

In the new equation it becomes

$$
\gamma \alpha_{\lambda, \mu} \frac{y^{n+1-\lambda}}{x^{r-\mu}}
$$

As no denominator in $g(x)$ can be of degree greater than $r$ we have

$$
\mu \equiv 0
$$

$$
\begin{array}{lc}
\text { Also } & \alpha_{n+1-\lambda, r-\mu}=\gamma \alpha_{\lambda, \mu} \\
\mu<r_{n+1-\lambda} \\
& r-\mu<r_{\lambda} \\
\text { Hence setting } & \rho_{\lambda}=r-r_{\lambda}
\end{array}
$$

we see the exponents $\mu$ lie within the narrow interval defined by

$$
\rho_{\lambda} \leqq \mu \leqq r_{n+1-\lambda}
$$

For example for $n=11$ we find by $\S 3$

\begin{tabular}{c|rrrrrrrrrrr}
$\lambda$ & 11 & 10 & 9 & 8 & 7 & 6 & 5 & 4 & 3 & 2 & 1 \\
\hline$\rho_{\lambda}$ & 0 & 2 & 3 & 5 & 6 & 7 & 9 & 10 & 11 & 13 & 14 \\
$r_{n+1-\lambda}$ & 1 & 2 & 4 & 5 & 6 & 8 & 9 & 10 & 12 & 13 & 15
\end{tabular}

The $T$ equation thus has the form for $n=11$,

$$
\begin{gathered}
y^{12}+\frac{a+b x}{x} y^{11}+\frac{c}{\chi^{2}} y^{10}+\frac{d+e x}{\chi^{4}} y^{9}+\frac{f}{\chi^{5}} y^{8}+\frac{g}{x^{6}} y^{7}+ \\
\frac{h+j x}{\chi^{8}} y^{6}-\frac{g}{\chi^{9}} y^{5}-\frac{f}{x^{10}} y^{4}-\frac{e+d x}{\chi^{12}} y^{3}-\frac{c y^{2}}{\chi^{13}} \\
-\frac{b+a x}{x^{15}} y-\frac{1}{\chi^{15}}=0
\end{gathered}
$$


7.

Still other relations between the coefficients we get on showing that $T=0$ remains unaltered for the substitution

$$
\left(\begin{array}{cc}
y & x \\
y^{-n} x^{-r} & x^{n} y^{8}
\end{array}\right)
$$

In fact in the identity

$$
T\left[y_{\infty}(\tau), x(\tau)\right]=0
$$

replace $\tau$ by $\frac{\tau}{n}$; it goes over into

$$
I\left[\gamma y_{0}^{-n} x^{-r}, \quad x^{n} y_{0}^{8}\right]=0
$$

Hence etc., as before. This shows that on applying (15) to $\left(2^{a}\right)$

$$
\alpha_{\lambda, \mu} \frac{y^{\lambda}}{\chi^{\mu}}
$$

goes over into

$$
\gamma^{\lambda+1} \alpha_{\lambda, \mu} \frac{y^{\lambda^{\prime}}}{\chi^{\mu^{\prime}}}
$$

in the new equation, where

$$
\begin{gathered}
\lambda^{\prime}=n(n+1-\lambda)-8 \mu \\
\mu^{\prime}=n \mu-(n-\lambda) r
\end{gathered}
$$

and hence the coefficient of $\frac{y^{\lambda^{\prime}}}{x^{\mu^{\prime}}}$ is

$$
\alpha_{\lambda^{\prime}, \mu^{\prime}}=\gamma^{\lambda+1} \alpha_{\lambda, \mu}
$$

For example for $n=11$ this shows that

$$
-\partial=a \quad j=-c \quad-c=-h
$$

This permits us to write (14) in the more precise form

$$
\begin{aligned}
& \text { (16) } y^{12}+\frac{(a+b x)}{x} y^{11}+\frac{c y^{10}}{\chi^{2}}+\frac{-a+e x}{x^{4}} y^{9}+\frac{f}{x^{5}} y^{8}+\frac{g y^{7}}{\chi^{6}}+ \\
& \frac{c-c x}{x^{8}} y^{6}-\frac{g y^{5}}{x^{9}}-\frac{f y^{4}}{x^{10}}+\frac{-e+a x}{x^{12}} y^{3}-\frac{c y^{2}}{x^{13}}-\frac{b+a x}{x^{15}} y-{ }_{x^{15}}^{1}=0
\end{aligned}
$$

As here

$$
T(y, 1)=(y-1)(y+1)^{11}=0
$$


we see that

$$
\begin{gathered}
a+b=10, \quad c=44, \quad-a+e=110 \\
f=165 \quad g=132
\end{gathered}
$$

Thus to get all the coefficients of (16) it is necessary to find one only of the three quantities $a, b, e$.

8.

This we get by remarking another property of our $T$ equation. In fact we saw $\& 2$ that for $x=0, y_{\infty}=\gamma 2^{-m}$ while all the other roots were infinite. If then we replace in $T=0, y$ by $y^{-1}$ all the roots of the resulting equation will be zero except one which will be $\gamma 2^{m}$. This gives at once

$$
r_{n}=r \quad a_{n, 0}=-2^{m}
$$

For $n=11$ this gives $a_{n, 0}=-b=-32$

hence, $a=-22 \quad b=32 \quad e=88$ and our equation assumes its final form

$$
\begin{gathered}
y^{12}+\frac{-22+32 x}{x} y^{11}+\frac{44}{\chi^{2}} y^{10}+\frac{22+88 x}{x^{4}} y^{9}+\frac{165}{x^{5}} y^{8}+ \\
\frac{132}{x^{6}} y^{7}+\frac{44(1-x)}{x^{8}} y^{6}-\frac{132}{\chi^{9}} y^{5}-\frac{165}{x^{10}} y^{4}-\frac{88+22 x}{x^{12}} y^{3}- \\
\frac{44}{\chi^{13}} y^{2}-\frac{32-22 x}{\chi^{15}} y-\frac{1}{x^{15}}=0
\end{gathered}
$$

For primes > 11 we must have recourse to the method indicated by Sohnke and referred to $\$ 2$, to obtain all the coefficients.

9

In conclusion let us look back and compare the $T$ equation with the usual modular equation. We observe that whereas the modular equation in the usual theory occupies a position apart requiring a separate treatment, the $T$ equation is only one of a great family of equations, the $W$ equations. If we deduct Arts. 1 and 5 which concern the rationality of the coefficients and the irreducibility and which pertain to all the equations of this class, we see the theory of our $T$ equation reduces to very small dimensions. Finally we pass from $T=0$ to $M=0$ on setting $y=u^{-n} v$. These three counts seem to make it desirable to replace the 
historical modular equation by the $I$ equation which may be regarded as the modular equation suitably normed from our present standpoint.

If we desire an equation whose roots like those of the modular equations remain finite for finite $x$, we should replace the $T$ equation by the equation $U(z, x)=0$ generated by

$$
z_{\infty}=\Pi \frac{d n}{c n}\left(p \frac{4 K}{n}\right)=y_{\infty}^{-1} . \quad p=1,2 \cdots m
$$

For $n=11$ such an equation is got from (17) by replacing $y$ by $y^{-1}$, we have then

$$
\begin{gathered}
z^{12}+(32-22 x) y^{11}+44 x^{2} z^{10}+(88+22 x) x^{3} z^{9}+165 x^{5} z^{8} \\
+132 x^{6} z^{7}-44(1-x) x^{7} z^{6}-132 x^{9} z^{5}-165 x^{10} z^{4}-(22+88 x) x^{11} z^{3} \\
-44 x^{13} z^{2}+(22-32 x) x^{14} z-x^{15}=0 .
\end{gathered}
$$

The theory of these equations can, of course, be made independent of the $T$ equations.

New Haven, Conn.,

March, 1897.

\section{CORRECTION.}

The following errata occur in the abstract of Professor Felix Klein's Princeton Lecture "On the Stability of a Sleeping Top," printed in the January number of the BuLLETIN, pp. 129-132:

At the bottom of page 130, read

$$
U=2(u-1)\left(n^{2}+(P u-h)(\dot{u}+1)\right) .
$$

In the middle of p. 131, read

$$
v^{2}=\frac{(1-e)\left(n^{2}-2 P(e+1)\right)}{e+1} .
$$

On page 132 , fifth line from the bottom, read $n^{2}-4 P$ instead of $u^{2}-4 P$. 